\title{
TRES CONSIDERACIONES SOBRE GESTIÓN UNIVERSITARIA QUE PERMITEN UN CAMBIO EN EL PARADIGMA ORGANIZACIONAL
}

\section{Introducción}

La Universidad Arturo Prat (UNAP) es una de las 16 universidades estatales que conforma, junto a nueve universidades privadas, el grupo de universidades tradicionales representadas a través del Consejo de Rectores de las Universidades Chilenas.

Esta casa de estudios nace el año 1984, como uno de los productos del proceso de reorganización del sistema universitario nacional, dentro del marco de la creación de las universidades regionales. De esta forma, la actual UNAP aparece como continuadora legal del Instituto Profesional de Iquique y éste, a su vez, de la Universidad de Chile sede Iquique.

En el momento de su nacimiento, la universidad se caracterizaba por tener una escasa y débil infraestructura física, carreras muy poco atractivas para la población estudiantil local y heredar, como producto del decreto con fuerza de ley $\mathrm{N}^{\circ} 4$ del 14 de enero de 1981 del Ministerio de Educación, un exiguo aporte de recursos económicos provenientes del Estado (aporte fiscal directo de la época: 0,47\% del total a repartir entre las universidades).

Todas estas características iniciales, en conocimiento del cuerpo académico, actuaron favorablemente para generar todo un proceso de desarrollo destinado a cambiar su condición de instituto por el de universidad, permitiendo construir una cultura organizacional. Como pretendo demostrar en este artículo, tres elementos han sido fundamentales para crear un modelo de administración universitaria que ha permitido transformar esta pequeña 
sede de la Universidad de Chile en una institución que hoy se desarrolla con vigor.

\section{El primer elemento de gestión: un organigrama} plano, con unidades de gestión pequeñas, pero con alto nivel de decisión (unidades pequeñas con "empoderamiento")

Nadie puede desconocer que, hoy en día, la velocidad para tomar decisiones y operar es un factor relevante para el éxito de las organizaciones. Un punto clave para dar agilidad a la universidad es la construcción de los niveles jerárquicos en la organización. En este cuadro de mando se debe lograr una estructura organizacional que tenga al menos dos características: por una parte, que todos aquéllos que participan en la organización tengan el mayor poder de decisión lo más cercano posible, evitando la burocracia o la pérdida de energía por el traspaso entre eslabones; por otra, que exista un nivel de decisión o "empoderamiento" importante en la propia unidad operacional de donde nacen las iniciativas que permiten generar nuevas acciones en la organización.

Para su quehacer en docencia, investigación y extensión, la universidad posee una estructura organizacional basada en unidades académicas. Su estructura básica la conforman los departamentos, institutos y escuelas, y, con otra complejidad, las sedes y centros universitarios ubicados en distintas regiones del país.

Si bien el marco estatutario que mantiene la UNAP desde su origen está diseñado con los parámetros típicos de una universidad tradicional, es decir, organizada a través de facultades y vicerrectorías, ello permite que haya encontrado la forma para mantener hoy un organigrama muy plano en que las unidades operativas principales sean sólo departamentos, escuelas e institutos (véase la figura 1). 
Un organigrama "aplanado" trabaja igual que lo hace una cadena alimenticia en la naturaleza. Esto es, mientras menos eslabones existan, mayor es la eficiencia. Lo anterior, porque el traspaso de energía entre eslabón y eslabón genera pérdidas ya sea por respiración o excreción. En una estructura organizacional esto podría interpretarse como pérdida de tiempo y energía por pasos sucesivos de la tareas entre los diferentes niveles de organización, sin incorporación de valor a la función. En otras palabras, sólo incremento de burocracia y gasto.

El resultado es interesante, porque cada una de las unidades operativas (departamentos, escuelas o institutos) tiene radicada en ella misma, además, una serie de decisiones operativas. Son pequeñas unidades formadas por un número de académicos que va desde cinco a 23 con jornada completa, más otros de media jornada y por horas; cuentan, además, con secretarias y un oficial administrativo contable, que tiene la misión de operar cuentas corrientes, permitiendo agilidad operativa, por ejemplo, en la entrega de pasajes y viáticos, adquisición de materiales y pagos de contratos a honorarios.

En los departamentos y escuelas radica también la administración de la o las carreras que allí residen; también se controla la prestación de servicios docentes a otras unidades académicas, como la administración de proyectos de extensión e investigación en las que intervengan académicos de esa unidad. En los institutos el modelo sigue el mismo patrón, pero para la administración de proyectos de investigación y posgrados.

No obstante, es importante consignar que todos los ingresos a la corporación se encuentran centralizados y que la operación financiera de los departamentos, escuelas e institutos se produce de acuerdo con un presupuesto de caja debidamente aprobado, lo que facilita la administración central en el flujo de recursos e información y la descentralización de la toma de decisiones. 
La creación de sedes, que aparecen desde 1991, también responde a este modelo de unidades operacionales "livianas", que permiten mantener las condiciones de gestión antes indicada, no obstante administrar algunas de ellas a un número importante de estudiantes (más de 2.000 alumnos).

El jefe de sede cumple igual función que un director de departamento, contando esta unidad de gestión con un nivel de organización mayor, al incorporar actividades como administración de laboratorios, sistemas de control docente y bibliotecas. En un estado de madurez mayor, las sedes incorporan también una unidad de finanzas, contabilidad y administración de los recursos humanos; este es el caso de la Sede Victoria de nuestra casa de estudios, que actúa como un centro de consolidación de la gestión que la universidad desarrolla en el sur del país (desde Santiago hasta Ancud), lo que justifica que la autoridad máxima tenga, en este caso, el rango de vicerrector de sede.

Ocupando este modelo de construir unidades operacionales livianas y con un importante "empoderamiento", la UNAP se ha ido expandiendo hacia otras ciudades llegando a conformar la estructura de gestión que se muestra en la figura 2. Como se desprende de ésta, la universidad cuenta con dos centros de consolidación y administración de los recursos financieros, uno es la Casa Central, en la ciudad de Iquique, y el otro la Sede Victoria, en la IX Región de la Araucanía. En el primero se reciben y consolidan los recursos económicos generados en nuestras unidades operacionales que abarcan desde Arica hasta la comuna de Santiago. En la sede Victoria, en cambio, se administran los recursos que se generan desde Ancud hasta las comunas de la Región Metropolitana, exceptuando Santiago. En esta última sede ocupan un lugar preponderante los centros asociados al Centro de Educación y Capacitación a Distancia (CECAD).

Estos dos núcleos de administración financiera asumen la responsabilidad de proveer los recursos necesarios para la operación, 
inversiones y desarrollo de las unidades académicas bajo su cargo. No obstante, toda la contabilidad general y los excedentes financieros se administran finalmente en la Casa Central en Iquique.

Entonces, podemos concluir -por ahora- que la UNAP tiene una organización estructurada en unidades operacionales pequeñas, con alta capacidad de gestión por el "empoderamiento" que ellas poseen, pero con un fuerte control sobre los recursos económicos, los que se encuentran administrados, en último término, por dos unidades de control de gestión financiera, como son la Casa Central en Iquique, por el norte, y la Sede Victoria, por el sur del país.

\section{El segundo elemento de gestión: la "reinvención" permanente}

El crecimiento de la UNAP se debe, principalmente, a que ostenta una cultura organizacional que da libertad e incentiva la creatividad entre sus miembros, ya sea por un buen nivel de tolerancia al error y al fracaso en la implementación de algunas iniciativas, o por la entrega de estímulos económicos a los creadores de nuevos proyectos.

En este marco, la UNAP se ha caracterizado por "reinventarse constantemente", fenómeno que se ha dado en dos dimensiones: por una parte, actuando sobre la operación rutinaria, es decir, cambiando lo que ha hecho por años; por otra, en la ampliación de la operación, la creación de nuevas carreras más allá de la oferta de pregrado, o nuevas acciones de extensión, gestión o creación de instancias cooperadoras con la universidad.

En lo rutinario, la UNAP ha llevado adelante importantes cambios tanto en la docencia como en la gestión. En docencia cabe mencionar, por ejemplo, la disminución de la duración de la hora de clase a 60 minutos, permitiendo agregar horas de clases "verdes" para cada una de las asignaturas, en donde el profesor tiene la libertad para desarrollarlas en la empresa, como talleres u otra 
modalidad que él quiera emplear. Otro cambio importante fue la eliminación de la cátedra de inglés técnico en la universidad, optando por incorporar posteriormente cinco trimestres obligatorios de inglés conversacional para todas las carreras. En el área de gestión se creó la dirección superior de administración del conocimiento, orientada a la búsqueda de recursos económicos a través del uso práctico del saber. En esta dirección se encuentran los servicios externos de consultorías, oferta de capacitación y la creación y administración de empresas con que hoy cuenta la corporación, y que permiten transferir los resultados de la investigación y la producción de nuevas tecnologías.

En el ámbito de la ampliación de la operación, la UNAP fue la primera universidad tradicional que, después de la reforma de 1984, reimpulsó acciones tales como ofrecer carreras técnicas vespertinas para trabajadores, salir de sus campus con planes especiales de continuidad de estudios y de ingresos para trabajadores, y crear sedes. También ha tenido la capacidad para diseñar y operar una unidad académica que desarrolla programas docentes con una modalidad muy particular de educación a distancia, la que ha resultado tremendamente exitosa. En este mismo aspecto, la universidad ha creado corporaciones, fundaciones y sociedades anónimas, que le permiten contar hoy con las defensorías penales públicas de las ciudades de Iquique, Antofagasta, Calama, Victoria y Angol; administrar dos parvularios, un centro de formación de operadores mineros (Centro Tecnológico Minero, CMT), tener un Instituto de Investigación Aplicada en el área de la acuicultura en Puerto Montt y participar en el control de gestión de un fundo productor de carne y una lechería en el sur de Chile.

Este proceso de reinvención permanente ha hecho posible que la universidad pueda crecer y consolidarse, ya que se ha obtenido una buena cantidad de recursos económicos y, con ello, se ha facilitado la inversión en recursos humanos capacitados (o la capacitación de los existentes en un mejor nivel) y la adquisición de equipamiento e infraestructura. 
En la figura 3 se esquematiza el proceso antes descrito. Cada "invención" es capaz de colaborar con un nivel de crecimiento hasta que alcanza un estrato y exige entonces una nueva invención. Los parámetros graficados en la abcisa corresponden a tiempos determinados que van desde el año 1989 en adelante.

\section{El tercer elemento de gestión: el modelo o fórmula del crecimiento}

En la figura 4 se muestra un esquema del modelo de expansión y crecimiento que ha llevado a cabo la UNAP. Éste tiene un comportamiento parecido al desarrollo de los niveles de organización biológica: célula, agrupación de células, tejidos, órganos y organismos.

Efectivamente, la universidad ha llevado a cabo su expansión territorial a través de la implantación de un programa docente inicial que nace desde un departamento o escuela ubicado en la casa central. En la figura 4 este tipo de acción se esquematiza con las letras A a F. Normalmente éste se instala por petición o por convenio con alguna organización social (colegio profesional, organización sindical o municipalidad).

Si el programa inicial o "célula germinal" tiene éxito-considerando como criterios la capacidad de matricular sucesivas promociones de alumnos en cantidad suficiente para sobrellevar los costos y generar una utilidad equivalente al 30\% en promedio- motiva entonces a que otras unidades puedan instalar también programas académicos en la misma ciudad, sin que necesariamente, en una primera etapa, se impartan todos ellos en el mismo lugar. Este modelo se repite con la participación de otras unidades académicas y determinando -en conjunto los departamentos, escuelas y la dirección superior de la UNAP- la conveniencia de congregar todos estos programas docentes en un solo espacio, con el fin de hacer más eficaz y eficiente su labor. 
Si el sistema de convergencia primario se torna más complejo, se requiere generar entonces una unidad con mayor nivel de organización, y se constituye un centro, el que cuenta con un director y mayor cantidad de personal administrativo. Todos ellos actúan coordinadamente, tanto para atender las carreras como para llevar adelante la administración de una unidad de documentación y la administración de laboratorios de computación en un primer estado de organización especializada, es decir, como un símil de organización de tejido celular.

Si el volumen de programas y alumnos aumenta en un lugar determinado, entonces el centro se transforma en sede universitaria, y allí el nivel de complejidad organizacional es mayor, ya que para asegurar una gestión de calidad se requiere una mayor autonomía administrativa y descentralización de la gestión financiera y de la administración académica. Se incorporan académicos de jornada completa que pertenecen a la sede. Este nivel de organización encuentra su símil en la biología de lo que es un órgano.

No obstante todo lo anterior, es importante indicar que ciertos programas especiales pueden frustrarse y deben desaparecer en el corto plazo, una vez que terminen con la última promoción de alumnos matriculados. En la figura 4 este fenómeno está representado con los círculos rotulados como F. Esta falta de éxito puede producirse porque no hay interés por el programa o porque sus condiciones no fueron las adecuadas para el mercado al cual estaba dirigido, entiéndase esto último como incompatibilidad de horarios con el trabajo, defectos en la orientación de los programas, entre otros factores.

La complejidad mayor del crecimiento organizacional se alcanza cuando ya la sede tiene una dotación importante de personal de jornada completa -académicos y administrativos- y se inicia el desarrollo de otras actividades, además de la docencia y extensión: aparecen la investigación y la oferta de servicios; se transforma este nivel organizacional en otra unidad generadora de nuevas 
acciones, que implican también expansión y crecimiento. En la UNAP esto se da actualmente con la Sede Santiago y Victoria. Especialmente se debe destacar esta última, que ha tenido la capacidad de crear unidades académicas voluminosas como el CECAD, con más de 10.000 alumnos a la fecha, y el Instituto de Ciencia y Tecnología en Puerto Montt, con más de 12 investigadores de jornada completa, que llevan adelante una serie de proyectos aplicados en el área de las ciencias marinas, con financiamientos del Fondo de Fomento al Desarrollo Científico y Tecnológico (FONDEF) y otras agencias nacionales e internacionales.

Otro hecho importante es que en la cultura organizacional de la UNAP existe una conciencia colectiva entre los académicos para estar atentos y aprovechar las oportunidades que se presentan y permitir el crecimiento y desarrollo de la corporación. Un ejemplo se refleja en la figura 4 donde se da cuenta de la creación de la sede Victoria el año 1991, que nació de la instalación de una unidad académica y administrativa con un nivel de organización intermedio, en que desde su origen se contó con un directivo superior y un grupo de académicos y administrativos que fueron trasladados desde la casa central para el desarrollo de esta nueva sede.

La oportunidad se presentó a través del ofrecimiento en comodato por 40 años -por parte de la municipalidad y la comunidad de la ciudad de Victoria- de un inmueble de $7.500 \mathrm{~m}^{2}$, en donde había funcionado la Escuela Normal y, posteriormente, la Pontificia Universidad Católica de Chile, con la finalidad de restituir la actividad universitaria a la comuna y poder entregar también educación superior a los habitantes de la provincia de Malleco. Esta oferta se evaluó y se decidió implementarla, en una época en que, prácticamente, no existían sedes de universidades tradicionales y las universidades particulares estaban recién emergiendo. Hoy esta sede tiene la responsabilidad de desarrollo y control de la gestión de varias unidades operativas y de más de 15.000 alumnos. 
Figura 1. Organigrama de la Universidad Arturo Prat.

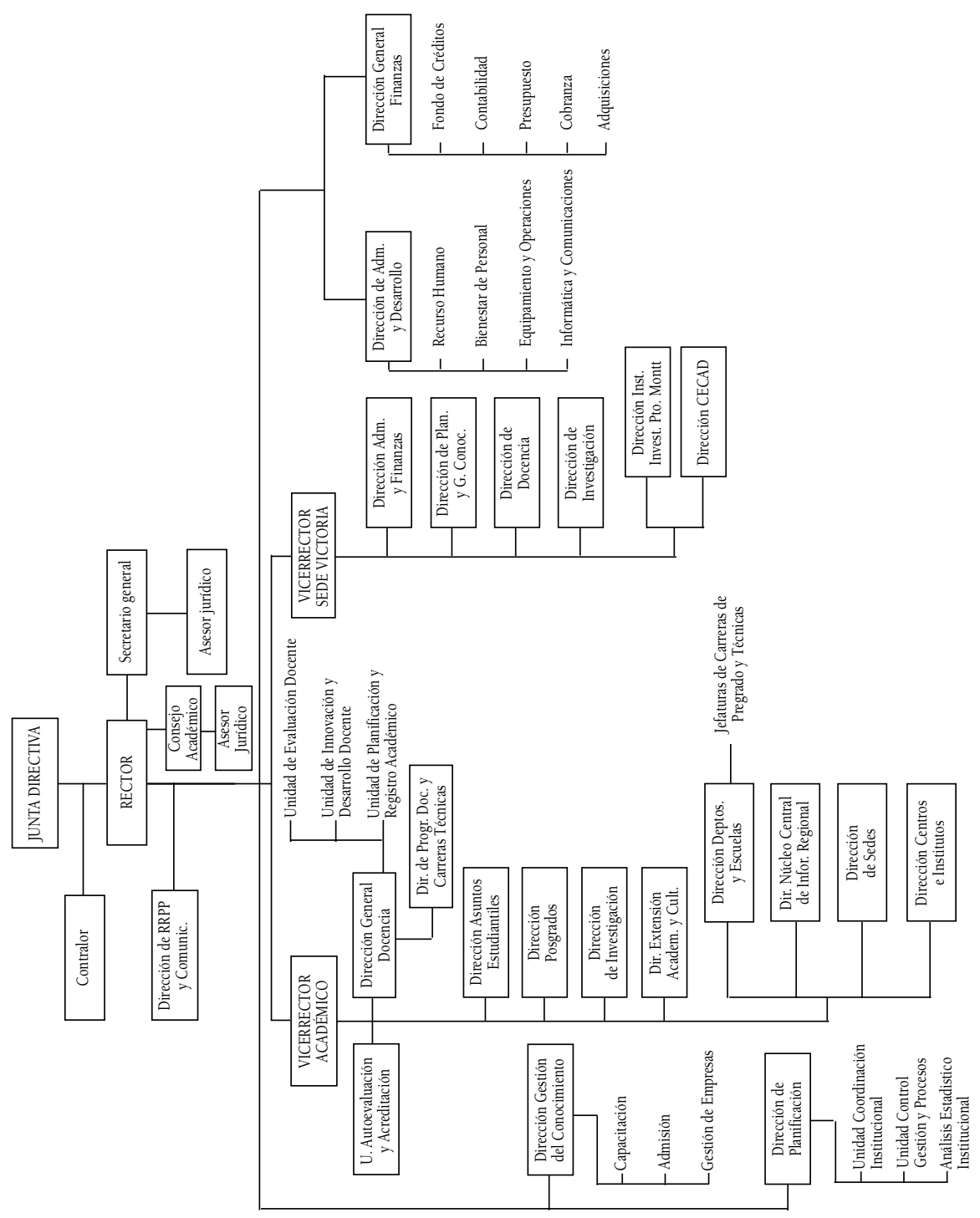


Figura 2. Estructura de gestión organizacional de la Universidad Arturo Prat.

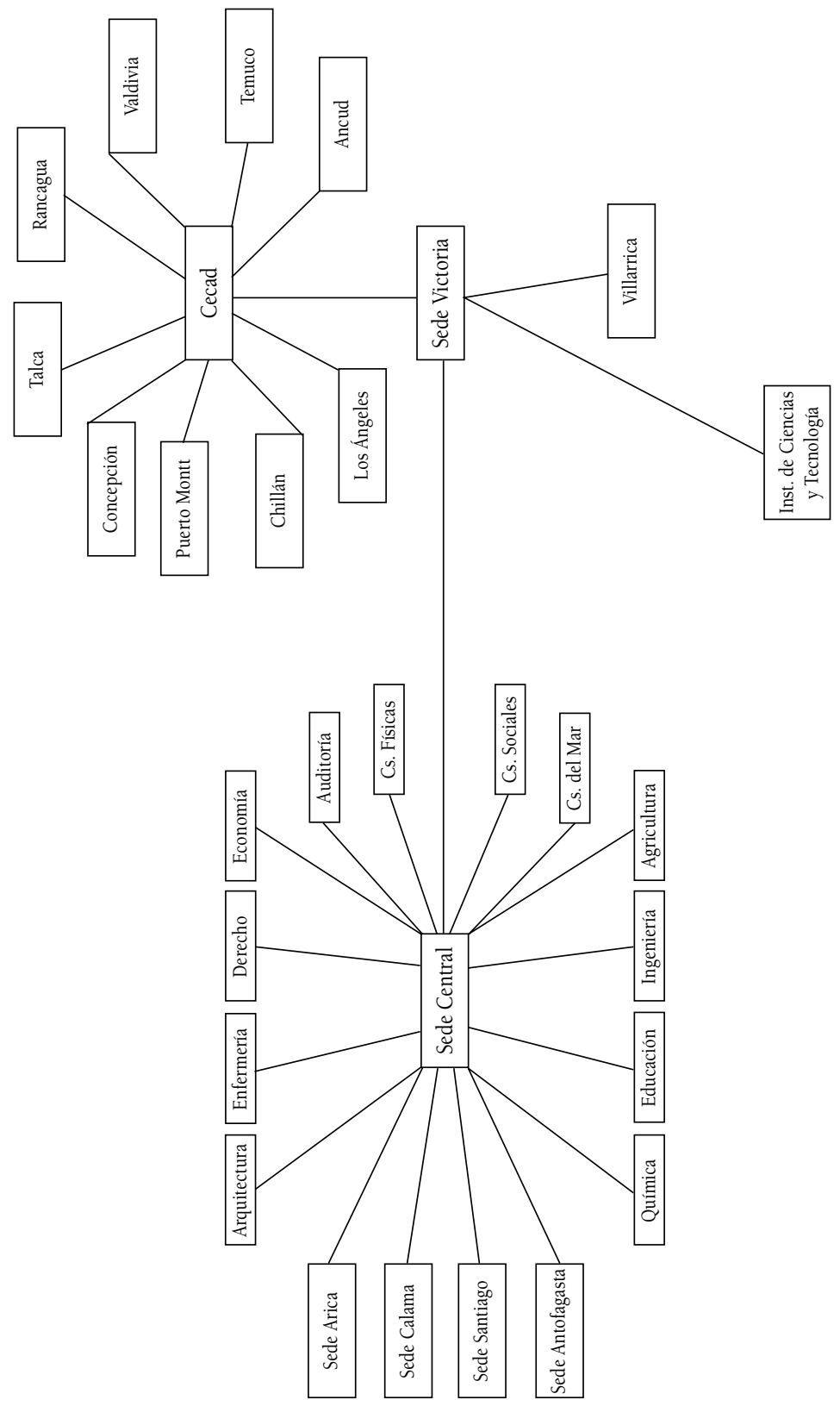


Figura 3. Evolución del proceso de consolidación y reinvención institucional.

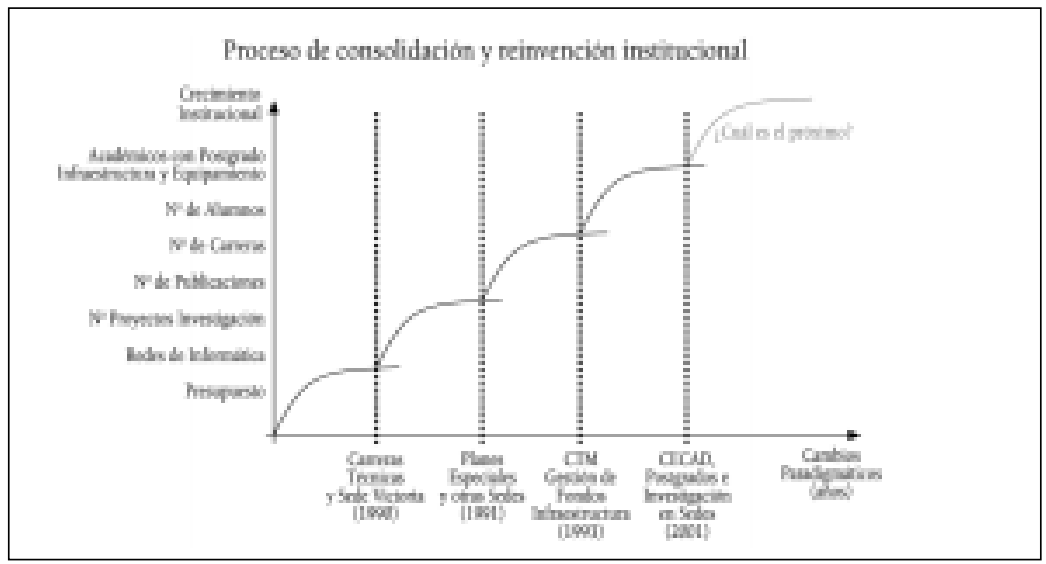

CTM = Centro Tecnológico Minero.

CECAD = Centro de Capacitación y Educación a Distancia.

Figura 4. Modelo de expansión y crecimiento de la Universidad Arturo Prat.

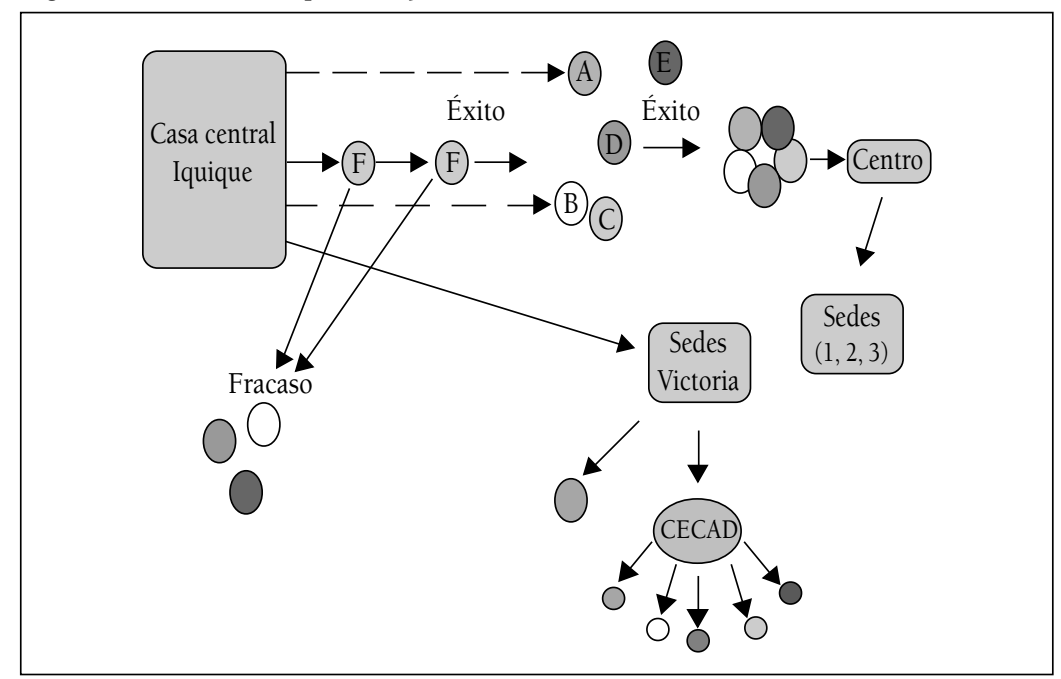

Sedes 1, 2, 3, 4 corresponden a cada una de las sedes en el Norte de Chile, y que nacen de acuerdo al modelo: 1. Sede Arica; 2. Sede Calama; 3: Sede Antofagasta; 4. Sede Santiago. 